\title{
Dimensionamiento y evaluación energética de un aerogenerador para instalar en una vivienda familiar
}

\section{Dimensioning and energy evaluation of a wind turbine installed in a family house}

Lidia del Rocío Castro Cepeda. ${ }^{1}$, Andrés Joao Noguera Cundar. ${ }^{2}$ \& Mónica Alexandra Moreno Barriga ${ }^{3}$

Abstract.

DOI https://doi.org/10.33262/cienciadigital.v3i4.2.1013

The present theoretical research study was conducted in the parish San Juan Chico,which objective is to calculate and design a wind turbine that can be attached to a single-family home in order to avoid the consumption of the network. A scientific methodology was applied to start from data that have been collected several years ago without giving them an investigative treatment; the techniques used to collect this data were to go to the database that ESPOCH has. Then we proceeded to calculate the characteristic wind curves through calculation tools that already exist for this type of wind turbines, and then the generator power and its construction elements were designed. Finally, the typical consumption of a single-family home was calculated and compared with the one that is going to be generated arriving at effective and efficient results with what can be expected that this theoretical work can become an experimental work and thus scientifically prove the Results obtained during this investigation.

Keywords: Wind turbine, wind energy, renewable energy, electricity, wind.

\footnotetext{
${ }^{1}$ Escuela Superior Politécnica de Chimborazo, Facultad de Mecánica. Riobamba, Ecuador. lidia.castro@espoch.edu.ec

${ }^{2}$ Escuela Superior Politécnica de Chimborazo, Facultad de Mecánica. Riobamba, Ecuador. andres.noguera@espoch.edu.ec

${ }^{3}$ Escuela Superior Politécnica de Chimborazo, Facultad de Mecánica. Riobamba, Ecuador. monica.moreno@espoch.edu.ec
} 


\section{Resumen.}

El presente estudio teórico de investigación se realizó en la parroquia San Juan Chico, Cantón Calpi, Provincia de Chimborazo cuyo objetivo es calcular y diseñar un aerogenerador que se pueda acoplar a una vivienda unifamiliar con el fin de evitar el consumo de la red. Se aplicó una metodología científica para partir de datos que se han recolectado hace varios años sin darles un tratamiento investigativo; las técnicas utilizadas para recopilar estos datos fue acudir a base de datos con las que cuenta la ESPOCH. Luego se procedió a calcular las curvas características del viento a través herramientas de cálculo que ya existen para este tipo de aerogeneradores, posterior se diseñó la potencia del generador y sus elementos de construcción. Finalmente se calculó el consumo típico de una vivienda unifamiliar y se comparó con la energía que se espera generar con el equipo, llegando a resultados efectivos y eficientes. Esta investigación teórica puede convertirse en un trabajo experimental y de esta forma probar los resultados obtenidos.

Palabras claves: aerogenerador, energía eólica, energía renovable, electricidad, viento.

\section{Introducción.}

La energía renovable es un término utilizado ampliamente en la actualidad, hace referencia a todos los tipos de energía que provienen de fuentes limpias e inagotables, tienen gran potencial puesto que estas son: diversas, abundantes y cumplen con una condición muy beneficiosa para el medio ambiente que es la no emanación de gases de efecto invernadero, ni emisión de contaminantes.

Analizando las importantes características, es fácil entender el crecimiento imparable que se está observando en el aplicación y desarrollo de nuevas tecnologías que aprovechen de manera eficiente estas energías.

Dentro de las fuentes de energía importantes se encuentra la llamada "energía eólica", es aquella que tiene como fuente única al viento y de forma resumida se la puede definir como una energía cinética producida por consecuencia de las corrientes de aire, sus aplicaciones son variadas sin embargo se pueden clasificar en tres grupos que se derivan de la trasformación de la energía eólica en: energía mecánica, térmica y eléctrica, siendo esta última la que tiene más aplicación a nivel mundial.

Es importante entonces, enfocar el desarrollo de alternativas que aprovechen el viento y lo conviertan en energía eléctrica. Estas tecnologías en la actualidad ya se encuentran en marcha, su principal aplicación es la utilización de aerogeneradores, dispositivos que son capaces de transformar la energía cinética del viento en energía mecánica pues su estructura está formada por hélices diseñadas aerodinámicamente que bajo acción del viento producen 
un movimiento rotatorio, luego esta energía mecánica a través de un sistema de trasmisión por ejes hace girar un generador de corriente trifásica logrando de esta forma convertir la energía cinética en energía eléctrica.

Las distintas características de un aerogenerador como su potencia, tipo, disposición de las hélices, requerimientos de la red, etc, han hecho que la generación de electricidad eólica se aplique alrededor del mundo, principalmente en los conocidos parques eólicos o plantas de generación eléctrica eólica, en donde se encuentran emplazadas en campos abiertos de grandes áreas, varias torres de generación distanciadas unas de otras en función de condiciones como, velocidad del viento, movimiento de las hélices, impacto ambiental, etc.

Los países con más participación en producción de energía eléctrica mediante aerogeneradores son Estados Unidos, China, Alemania, España, India, Reino Unido, Canadá, Francia, entre otros, con la observación de que todos ellos utilizan únicamente grandes campos eólicos, es decir que los mismos son la principal fuente de electricidad. Con el análisis de esta importante característica, se convierte en una opción significativa escalar esta tecnología a los hogares, de tal forma que se logre una aplicación de la aerogeneración de corriente más común, es decir llevar estas técnicas de macro (campos eólicos) a micro (domicilios comunes). Esta forma de utilización de la energía del viento, podría entonces hacerse más visible y empezaría a ser más utilizada, logrando a largo plazo, por ejemplo, desplazar la utilización de combustibles fósiles por completo.

El presente trabajo pretende demostrar que la utilización de este tipo de generación eléctrica es posible en hogares, se realiza un análisis, cálculo, dimensionamiento y evaluación energética de un aerogenerador que se ubicaría en una vivienda unifamiliar en la comunidad de San Juan Chico a aproximadamente $20 \mathrm{~km}$ de la ciudad de Riobamba en la vía a Guaranda, tomando en cuenta parámetros como: características de la turbina eólica, características del generador, velocidad y frecuencia del viento, los resultados se contrastan con el consumo local y precios actuales, comprobándose la factibilidad de la utilización de energía eólica como fuente de energía eléctrica en hogares de la ciudad.

\section{Metodología}

Para la investigación realizada del dimensionamiento y la evaluación energética del aerogenerador, en base a los objetivos planteados se utilizó una metodología de tipo científico y descriptiva, por la naturaleza de los datos se aplicó los métodos cualitativo-cuantitativo, analítico-sintético, las técnicas usadas para recolectar la información primaria corresponden a los datos de viento de San Juan Chico, que sirven para determinar la velocidad del viento presente en un lugar y tiempo, además constituye el fundamento base para el dimensionamiento del aerogenerador. Por otro lado, se considera el análisis del consumo energético de una vivienda familiar que corresponde a la muestra estudiada, y finalmente se calcula cual sería la variación obtenida con la instalación del aerogenerador 


\section{Resultados y Discusión}

Para el diseño se parte las condiciones iniciales que se muestran a continuación:

\section{Condiciones de Diseño}

- Características de la Turbina Eólica.

1. Longitud de palas: $1 \mathrm{~m}$

2. Curva $\mathrm{Cp} / \lambda$

$$
\begin{gathered}
\mathrm{C}_{\mathrm{p}}(\lambda)=0,40 \cdot \operatorname{sen}\left(\pi \cdot \frac{\lambda}{\lambda_{\text {límite }}}\right)-0,15 \cdot \operatorname{sen}\left(2 \pi \cdot \frac{\lambda}{\lambda_{\text {límite }}}\right) \\
\text { Donde, } \ldots \lambda_{\text {límite }}=14
\end{gathered}
$$

- Características del Generador:

- Tipo: Trifásico inducción de Jaula doble bobinado

- $\quad$ Datos de acometida trifásica 220 V y $60 \mathrm{~Hz}$

\section{Proceso de Diseño}

Como primer paso para el dimensionamiento del aerogenerador se determina las curvas características del viento que representa el comportamiento de esta máquina eléctrica; en este caso se consideran las características de la turbina y generador, y es importante mencionar que para el diseño específico no se cuenta con la caja multiplicadora, lo que favorece al diseño ya que al ser de un tamaño aceptable $(1 \mathrm{~m})$ se podrá instalar sin mayores obras civiles extras a las de una vivienda que posea una terraza común.

Luego se determina los valores del coeficiente de potencia Cp para los diferentes valores de $\lambda$ que es la relación entre la velocidad tangencial de la punta de la pala del aerogenerador y la velocidad real del viento, para cada una de sus velocidades que se tendrá en el eje, dependiendo de las velocidades del viento que aproximadamente van desde $3 \mathrm{~m} / \mathrm{s}$ a $10 \mathrm{~m} / \mathrm{s}$.

De lo que se tiene

$$
\lambda=\frac{\Omega R}{V}
$$

Se calcula los diferentes valores que tendrá el coeficiente de velocidad en la punta de pala $[\lambda]$ a diferentes velocidades del viento, a través de la siguiente fórmula:

$$
C_{P}(\lambda)=0,40 \cdot \sin \left(\pi \cdot \frac{\lambda}{\lambda_{\text {lim }}}\right)-0,15 \cdot \sin \left(2 \pi \cdot \frac{\lambda}{\lambda_{\text {lim }}}\right)
$$

Luego se calcula los valores del coeficiente de potencia para las diferentes velocidades del viento (velocidad media anual del viento). Una vez que se tienen los datos anteriores se puede calcular el coeficiente de par [Cm]. 


$$
C_{m}(\lambda)=\frac{C_{P}(\lambda)}{\lambda}
$$

Se calcula el par y la potencia que proporciona la turbina con una densidad del aire de:

$$
\rho=1,29 \mathrm{~kg} / \mathrm{m}^{3} \text {. }
$$

Finalmente se calcula la familia de curvas POTENCIA [W] VS VELOCIDAD [ $\Omega$ ] (velocidad angular en el eje) para las distintas velocidades medias anuales del viento comprendido entre 3 y $10 \mathrm{~m} / \mathrm{s}$, que detalla en la figura 1 .

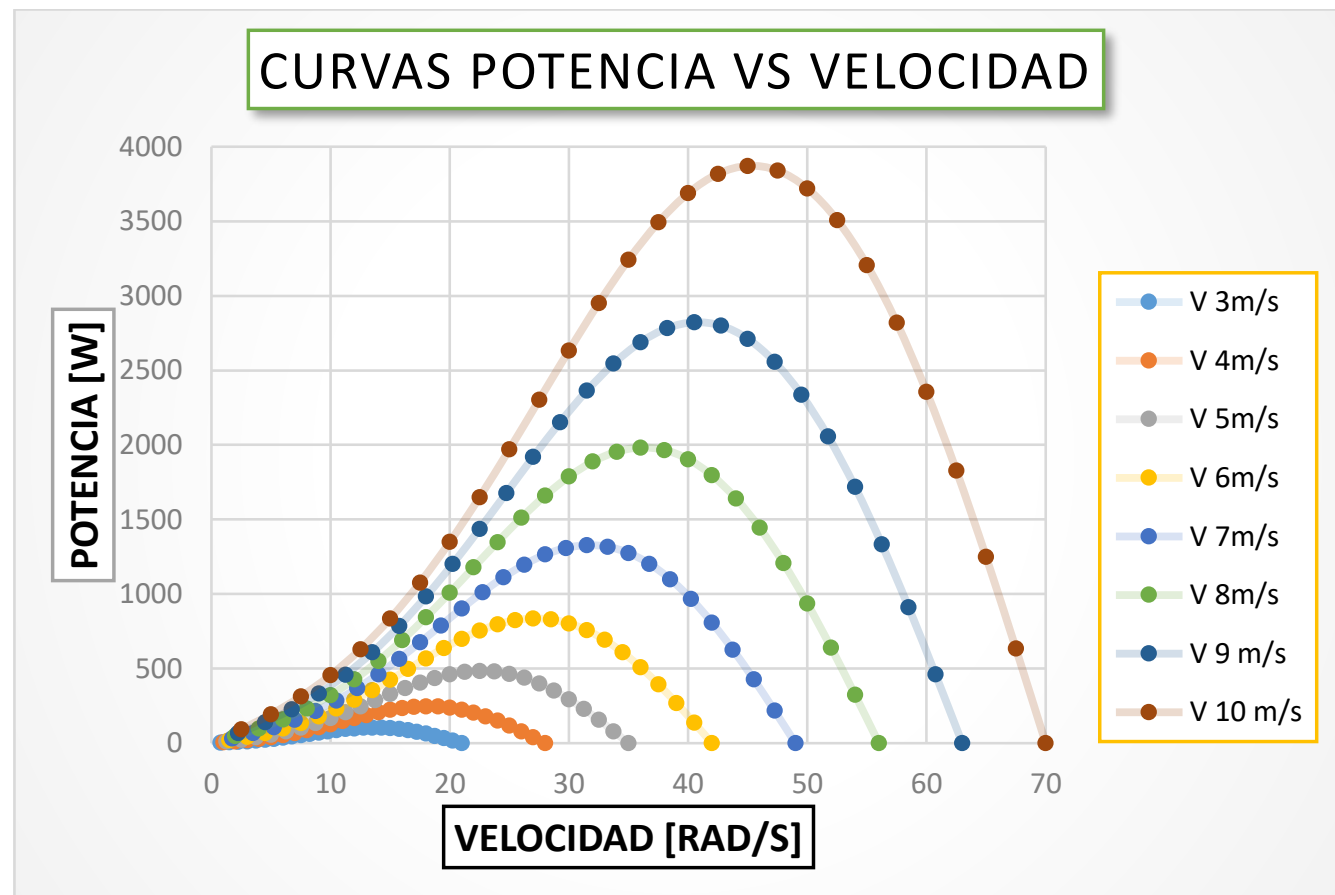

Figura 1. Familia de curvas POTENCIA VS VELOCIDAD DE LA TURBINA.

A partir de las curvas anteriores se grafica otra curva llamada PAR [M] VS VELOCIDAD $[\Omega]$, que corresponde a la figura 2 .

$$
T(V, \Omega)=\left[\frac{1}{2} \pi R^{3} \rho\right] \cdot V^{2} \cdot C_{m}(\lambda)
$$




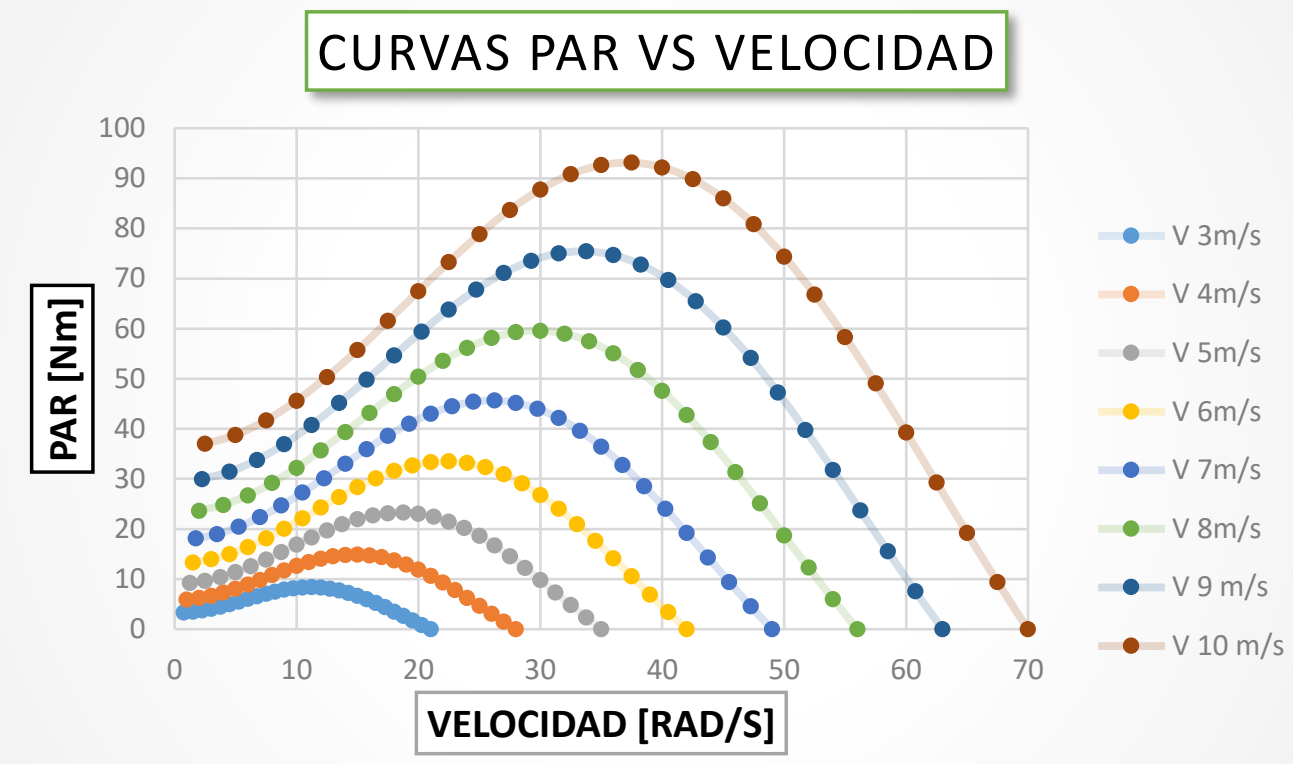

Figura 2. Familia de curvas PAR VS. VELOCIDAD de la turbina.

Para determinar las curvas par-velocidad para cada uno de los devanados del generador de inducción conectado a la red de acometida, se calcula el $[\lambda]$ óptimo a partir del coeficiente de potencia, que da como resultado:

$$
\begin{gathered}
\lambda_{\text {opt }}=\frac{\partial C_{P}(\lambda)}{\partial \lambda}=\frac{\cos ^{-1}\left(\frac{1}{6} \cdot(2-\sqrt{22})\right)}{\pi \cdot \lambda_{\lim }} \\
\lambda \text { optimo }=\mathbf{9 , 0 7 2 1 0}
\end{gathered}
$$

A través de esto se obtiene las gráficas de las curvas de los devanados contrapuestas a las de la turbina, que se representan en la figura 3. Se determina los devanados, cuyas características son el número de pares de polos y la potencia nominal, para ello se aplica el criterio de seleccionar el $\mathrm{Cm}[\lambda]$ y $\mathrm{Cp}[\lambda]$ óptimos, obteniendo como resultado los valores de la tabla 1:

\begin{tabular}{|c|c|}
\hline Cp $(\boldsymbol{\lambda}$ optimo) & 0,47777 \\
\hline $\mathbf{C m}(\boldsymbol{\lambda}$ optimo) & 0,05266 \\
\hline
\end{tabular}

Tabla 1. Cm y Cp óptimos

Con los valores óptimos se procede a calcular la potencia y velocidad de cada uno de los devanados a usar en el generador eléctrico, para el viento medio anual de nuestra aplicación:

\begin{tabular}{|c|c|}
\hline V media viento $[\mathbf{m} / \mathbf{s}]$ & 6 \\
\hline Frecuencia $[\mathbf{H z}]$ & 60 \\
\hline
\end{tabular}




\section{Voltaje [V] 220}

Tabla 2. Valores básicos para cálculos de velocidad y potencia.

Usando las fórmulas de Potencia y Par en la turbina, se encuentra que los valores más acertado para este diseño se representa en las tablas 3 y 4.

\begin{tabular}{|c|c|}
\hline$\Omega 1[R P M]$ & 150 \\
\hline$N^{\mathbf{o}}$ de Polos & 20 \\
\hline Potencia [W] & 550 \\
\hline Z base & 96,182 \\
\hline
\end{tabular}

Tabla 3. Valores nominales de Devanados de Baja.

\begin{tabular}{|c|c|}
\hline$\Omega 2$ [RPM] & 250 \\
\hline $\mathrm{N}^{0}$ de Polos & 12 \\
\hline Potencia [W] & 1750 \\
\hline $\mathrm{Z}$ base & 30,229 \\
\hline
\end{tabular}

Tabla 4. Valores nominales de Devanados de Alta.

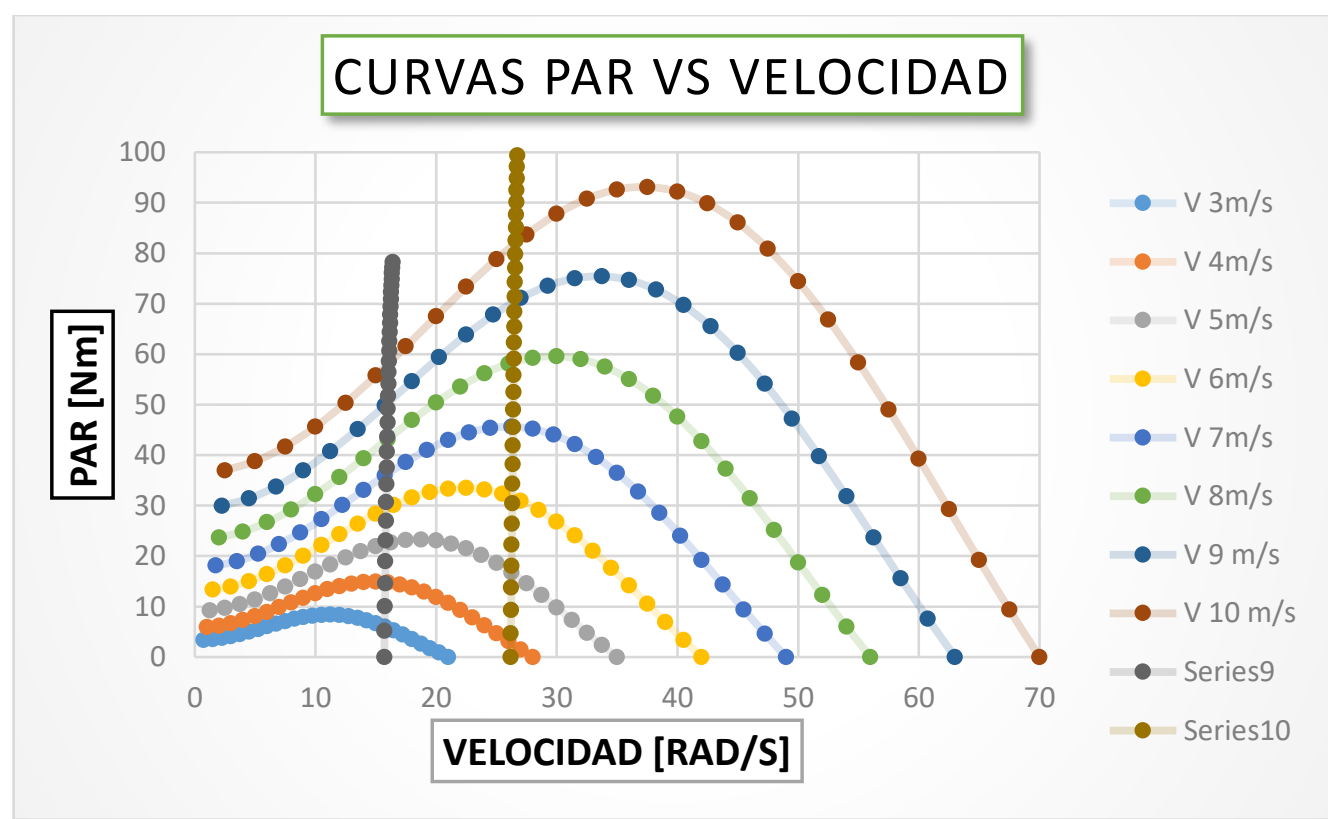

Figura 3. Curvas PAR VS. VELOCIDAD en contraparte con la potencia de los devanados 1 y 2 .

Las características y los valores de generación para los valores que tomará la velocidad del viento, se definen en un rango de operación de $3.5 \mathrm{~m} / \mathrm{s}$ a $6 \mathrm{~m} / \mathrm{s}$ donde entrará en funcionamiento el devanado de baja potencia con 20 pares de polos y 550 [W], mientras que 
para velocidades del viento superiores a $5 \mathrm{~m} / \mathrm{s}$ entrará en operación el devanado 2 de alta potencia de 12 pares de polos y 1750 [W].

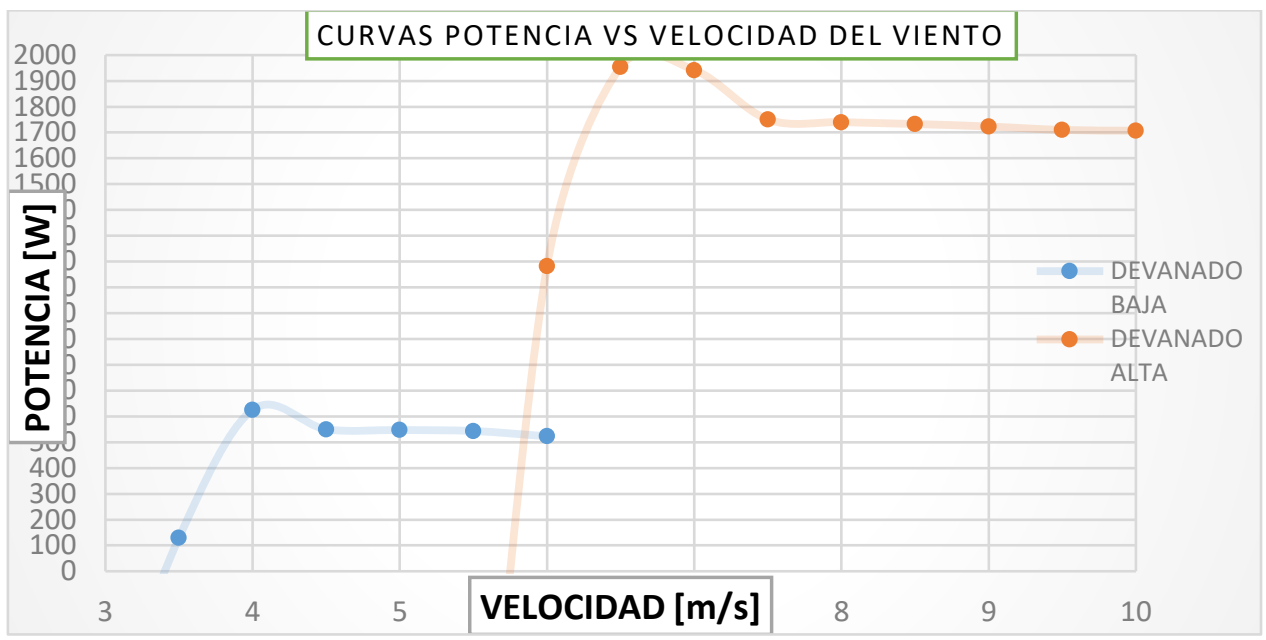

Figura 4. Gráficas de potencia nominales

En la figura 4 se observa las gráficas de potencia nominal con sus potencias netas (pérdidas) correspondientes a cada devanado, cuyo modo de conmutación entre los devanados de alta y baja potencia, para que el cambio se produzca de manera óptima cuando los valores de velocidad del viento fluctúen; será de $6 \mathrm{~m} / \mathrm{s}$ considerado éste como el punto de cambio entre devanados para lograr la mayor cantidad de energía generada y depender en menor medida del consumo de energía de la red.

A partir de ello se ha elegido el emplazamiento con mejores características de viento, que garantice la vida útil del aerogenerador y está situado en la provincia de Chimborazo, Cantón Calpi, parroquia San Juan Chico cuya velocidad media de viento anual es de $6 \mathrm{~m} / \mathrm{s}$ y a través de estos datos es posible obtener la distribución de Rayleig que se muestra en la figura 5.

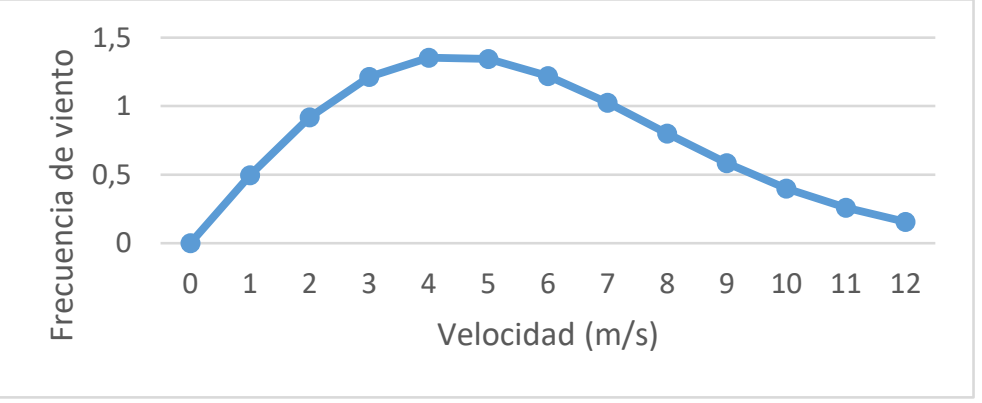

Figura 5. Distribución de Rayleigh para San Juan Chico

La figura 6 representa la potencia disponible a través de la velocidad media de viento que se tiene en el emplazamiento elegido que va desde $3 \mathrm{~m} / \mathrm{s}$ hasta $12 \mathrm{~m} / \mathrm{s}$ 


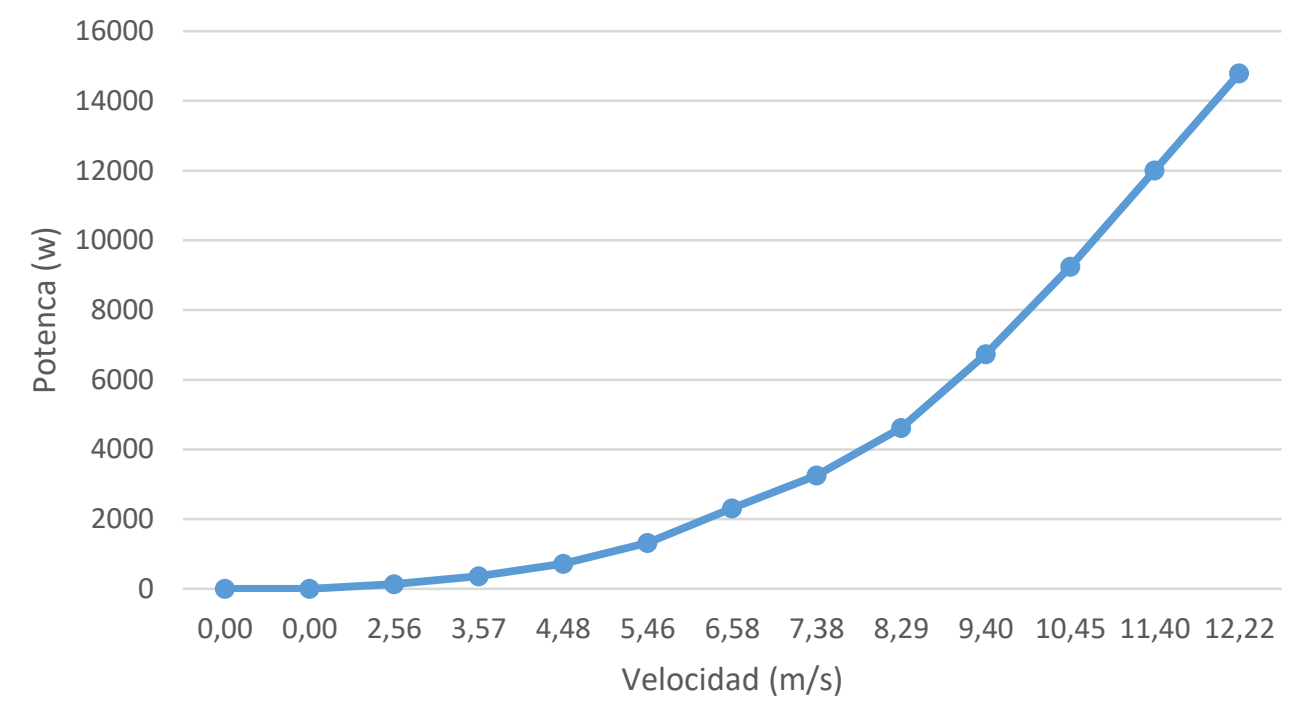

Figura 6. Viento - Potencia Disponible

Y finalmente se grafica la potencia anual generada dependiendo de la cantidad de viento que aproximadamente se genera durante todo el año, es importante aclarar que es un valor teórico calculado con los históricos de datos de viento obtenidos a través de una base de datos recogida por estudiantes de la Escuela Superior Politécnica de Chimborazo durante dos años antes.

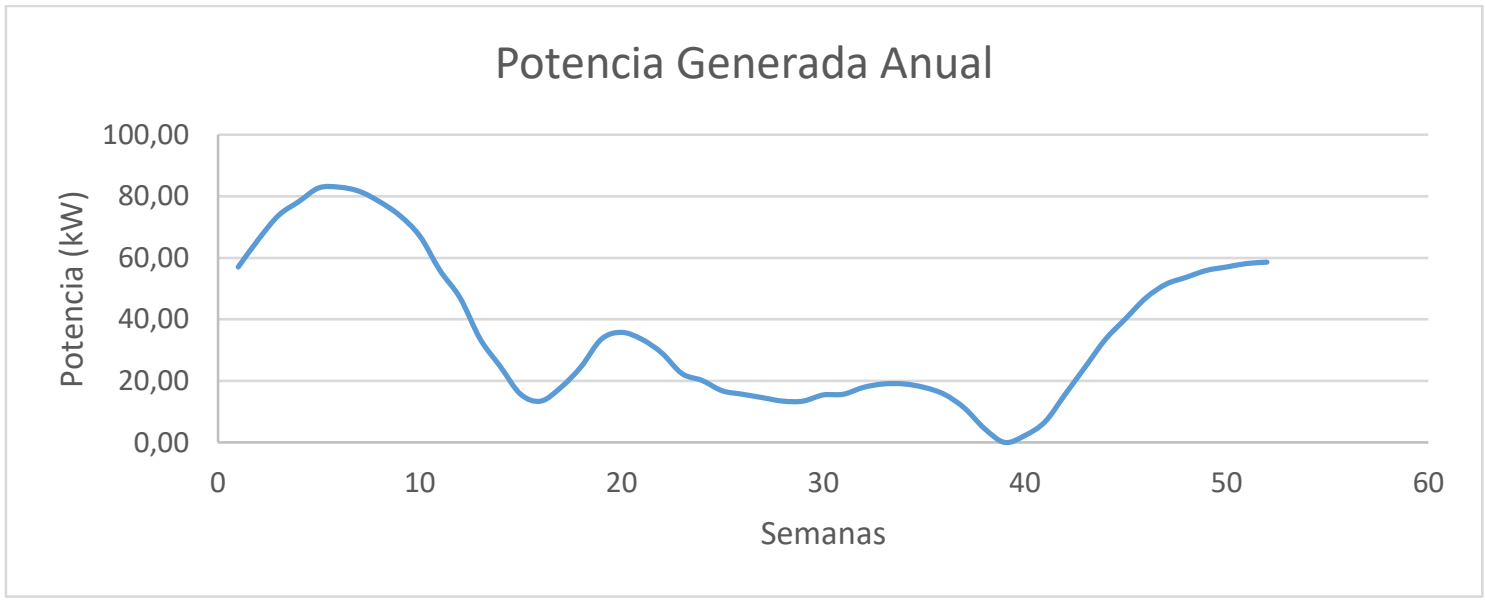

Figura 7. Potencia Generada Anual

Finalmente se debe calcular el consumo típico aproximado de una vivienda unifamiliar de tres habitantes con todos los servicios básicos incluida una ducha eléctrica, para de esta forma evitar el uso de un calefón que quema gas doméstico; de esta forma se evita quemar combustibles fósiles, mitigar la huella de carbono y la producción de gases de efecto invernadero. 
Dichos datos se resumen en la tabla 5 donde se detalla un consumo promedio por hora al día, sabiendo que en el Ecuador no existen estaciones se ha supuesto que todos los días se consume de la misma forma; para facilitar los cálculos, pero se considera cuatro días típicos uno por cada trimestre, en el que se utilice la gran mayoría de los equipos y electrodomésticos de consumo, es decir para los días con el mayor consumo, garantizando así que para el resto de días la energía producida sea mayor igual a la consumida, disminuyendo al máximo consumir la energía proveniente de la red.

\begin{tabular}{|c|c|c|c|c|}
\hline HORA & 1 & 2 & 3 & 4 \\
\hline 0 & 123,22 & 123,22 & 123,22 & 123,22 \\
\hline 1 & 123,22 & 123,22 & 123,22 & 123,22 \\
\hline 2 & 123,22 & 123,22 & 123,22 & 123,22 \\
\hline 3 & 123,22 & 135,22 & 123,22 & 123,22 \\
\hline 4 & 123,22 & 123,22 & 123,22 & 123,22 \\
\hline 5 & 123,22 & 123,22 & 123,22 & 123,22 \\
\hline 6 & 123,22 & 123,22 & 123,22 & 123,22 \\
\hline 7 & 455,12 & 155,12 & 155,12 & 155,12 \\
\hline 8 & 455,12 & 155,12 & 155,12 & 155,12 \\
\hline 9 & 1072,12 & 1002,12 & 252,12 & 552,12 \\
\hline 10 & 497,12 & 427,12 & 427,12 & 427,12 \\
\hline 11 & 1490,12 & 1120,12 & 1120,12 & 1120,12 \\
\hline 12 & 490,12 & 490,12 & 190,12 & 490,12 \\
\hline 13 & 1640,12 & 1570,12 & 1270,12 & 820,12 \\
\hline 14 & 420,12 & 420,12 & 420,12 & 120,12 \\
\hline 15 & 615,12 & 190,12 & 190,12 & 190,12 \\
\hline 16 & 120,12 & 120,12 & 120,12 & 120,12 \\
\hline 17 & 120,12 & 120,12 & 120,12 & 120,12 \\
\hline 18 & 120,12 & 120,12 & 120,12 & 120,12 \\
\hline 19 & 525,12 & 420,12 & 420,12 & 120,12 \\
\hline 20 & 504,12 & 434,12 & 434,12 & 434,12 \\
\hline 21 & 1100,12 & 995,12 & 695,12 & 570,12 \\
\hline 22 & 690,12 & 662,12 & 120,12 & 662,12 \\
\hline 23 & 662,12 & 662,12 & 127,12 & 427,12 \\
\hline
\end{tabular}

\section{Tabla 5. Consumo típico diario por periodo}

En la figura 8 se visualiza el consumo diario conectado a la red es decir un consumo normal y en naranja se visualiza el consumo con el aerogenerador, llegado a horas en el día en las que no utilizamos energía de la red, de esta manera se espera disminuir los costos mensuales y anuales de energía y sectorizar la producción de energía. 


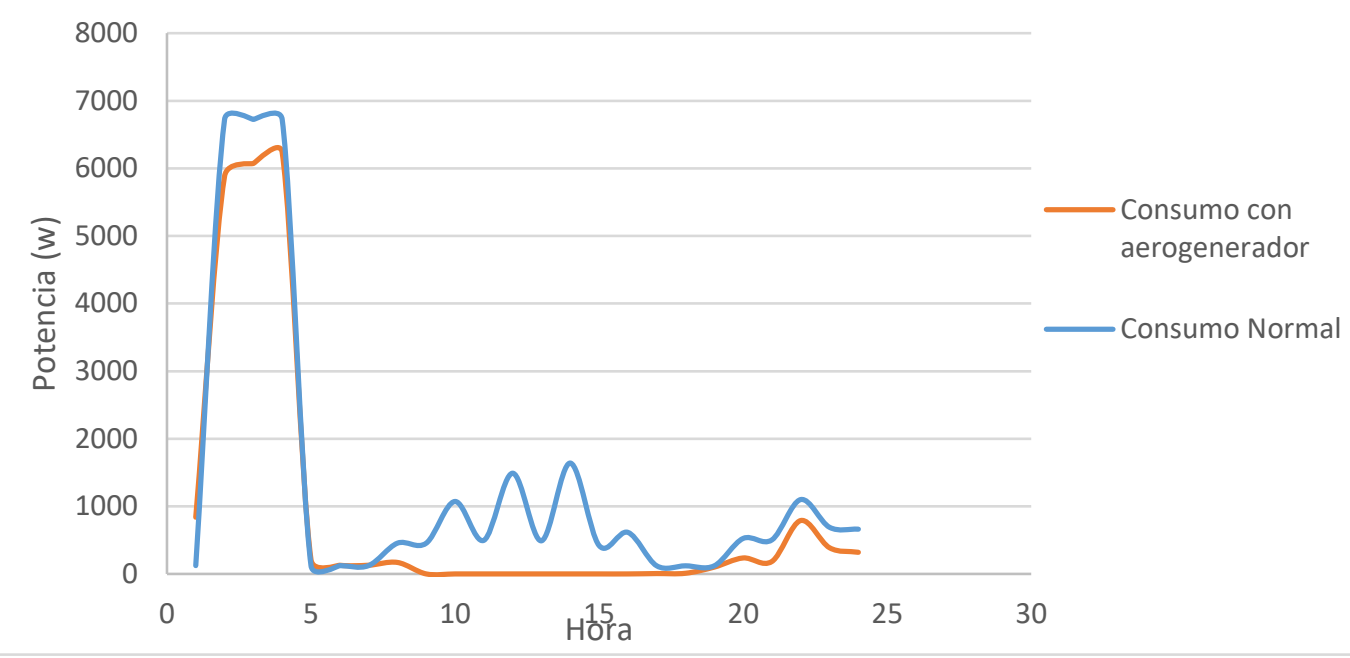

Figura 8. Consumo Horario

\section{Conclusiones.}

- Al instalar el aerogenerador se puede ahorrar alrededor del 50\% del costo anual de la energía consumida.

- El sistema se vuelve más rentable y eficiente pues se consume en gran medida lo que se genera, es así que se reduce la energía vertida de la red.

- La compra e instalación del aerogenerador conlleva una inversión, por lo que habría que estudiar el periodo de amortización para saber si compensa o no.

- No existe una normativa en el Ecuador para este tipo de aerogeneradores por lo que se complicará la instalación, lo ideal será utilizar un sistema autónomo de baterías.

- Al ser un pequeño aerogenerador con buenos resultados y con características especiales resultará comercial, una vez probado y con una fabricación en serie.

- Es conveniente realizar pruebas una vez instalado para comparar con otros tipos de generación renovable, como la energía solar fotovoltaica que ya tiene varias décadas con instalaciones en viviendas y ha dado buenos resultados.

- Con este tipo de estudios se puede incentivar a las autoridades locales y nacionales para invertir en proyectos innovadores y de esta manera disminuir la dependencia de los combustibles fósiles, que tanto daño causan al medio ambiente.

\section{Referencias Bibliográficas.}

- Hansen KS, Barthelmie RJ, Jensen LE, Sommer A. The impact of turbulence intensity and atmospheric stability on power deficits due to wind turbine wakes at Horns Rev wind farm. Wind Energy 2012;15:183. 
- Battisti, L., Benini, E., Brighenti, A., Dell'Anna, S., \& Raciti Castelli, M. (2018). Small wind turbine effectiveness in the urban environment. Renewable Energy, 129(Part A), 102-113.

- Bedon, G., De Betta, S., \& Benini, E. (2015). A computational assessment of the aerodynamic performance of a tilted Darrieus wind turbine. Journal of Wind Engineeringand Industrial Aerodynamics, 145, 263-269.

- Tabrizi, A. B., Whale, J., Lyons, T., \& Urmee, T. (2014). Performance and safety of rooftop wind turbines: Use of CFD to gain insight into inflow conditions. Renewable Energy, 67, 242-251.

- Dilimulati, A., Stathopoulos, T., \& Paraschivoiu, M. (2018). Wind turbine designs for urban applications: A case study of the shrouded diffuser casing for turbines. Journal of Wind Engineering and Industrial Aerodynamics, 175, 179-192.

- Hansen KS, Barthelmie RJ, Jensen LE, Sommer A. The impact of turbulence intensity and atmospheric stability on power deficits due to wind turbine wakes at Horns Rev wind farm. Wind Energy 2012;15:183.

- Samson, V., \& Paraschivoiu, M. (2018). Performance of a Darrieus turbine on the roof of a building. Transactions of the Canadian Society for Mechanical Engineering, 42(4).

- Pena A, Rathmann O. Atmospheric stability-dependent infinite wind-farm models and the wake-decay coefficient. Wind Energy 2014;17:1269.

- Celik, A. N. (2003). Energy output estimation for small-scale wind power generators using Weibull-representative wind data. Journal of Wind Engineering and Industrial Aerodynamics, 91(5), 693-707.

- P. M. O. Gebraad, J. W. van Wingerden, A Control-Oriented Dynamic Model for Wakes in Wind Plants, Journal of Physics: Conference Series 524 (2014) 012186, ISSN 1742-6596.

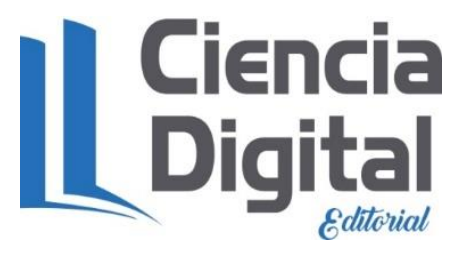




\section{PARA CITAR EL ARTÍCULO INDEXADO.}

Castro Cepeda, L. del R., Noguera Cundar, A., \& Moreno Barriga, M. (2019). Dimensionamiento y evaluación energética de un aerogenerador para instalar en una vivienda familiar. Ciencia Digital, 3(4.2), 116-128. https://doi.org/10.33262/cienciadigital.v3i4.2.1013

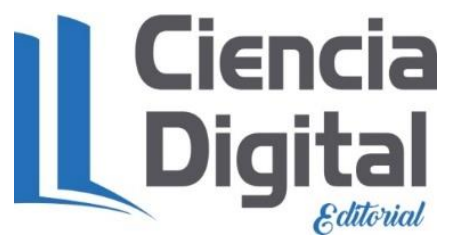

El artículo que se publica es de exclusiva responsabilidad de los autores y no necesariamente reflejan el pensamiento de la Revista Ciencia Digital.

El artículo queda en propiedad de la revista y, por tanto, su publicación parcial y/o total en otro medio tiene que ser autorizado por el director de la Revista Ciencia Digital.
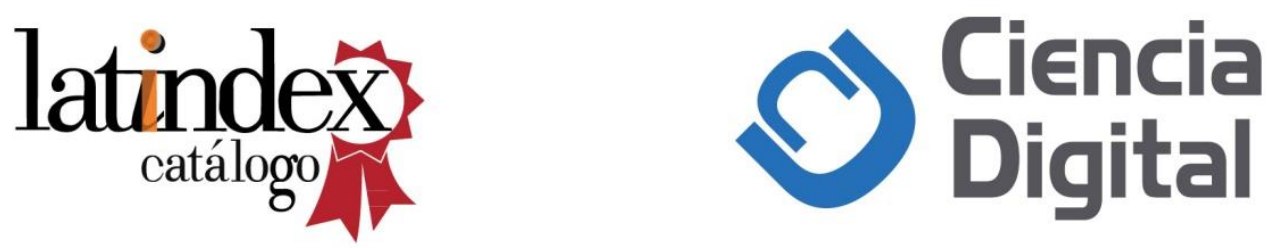